\title{
CAPACIDAD DE DEFORMACIÓN DE MUROS DE ALBAÑILERÍA CONFINADA PARA DISTINTOS NIVELES DE DESEMPEÑO
}

\author{
Maximiliano Astroza I. ${ }^{(1)}$ y Andrés Schmidt A. ${ }^{(2)}$
}

\begin{abstract}
RESUMEN
La construcción de viviendas de bajo costo, tanto en Chile como en el resto de los países latinoamericanos, se realiza principalmente en albañilería confinada. En general, se ha reconocido que este tipo de construcción ha tenido un buen comportamiento en los terremotos que han afectado la región. Con el propósito de contribuir al diseño sísmico de este tipo de edificios, en este trabajo se establecen los niveles de deformación asociados con diferentes estados límites, para lo cual se aprovecha la información de estudios experimentales de muros de albañilería confinada realizados en Chile, México y Venezuela.
\end{abstract}

\section{SUMMARY}

Confined masonry represents one of the most widely used construction systems in Latin America in dwellings built under government social programs and their seismic behavior have been good during the strong earthquake that affected the region. In order to contribute to the seismic design of this type of buildings, in this paper the deformation (drift) capacity of confined masonry walls for different limit states or performance levels is determined. With this objective, the results of some experimental studies developed in Chile, México and Venezuela have been used.

\section{INTRODUCCIÓN}

Las construcciones de albañilería confinada han sido muy usadas en Chile desde mediados del siglo pasado, especialmente por el buen desempeño observado en las viviendas de uno y dos pisos durante el terremoto de Chillán (Moya, 2002). Además ha contribuido a esta situación, el hecho que los materiales utilizados en su construcción sean relativamente económicos y que su ejecución no requiere de una inspección especializada, como ocurre con las albañilerías reforzadas con barras de acero distribuidas en el interior de los muros de albañilería.

La principal característica de este tipo de construcciones es que su sistema estructural está formado por muros como elementos primarios, los que deben soportar tanto las acciones de tipo gravitacional permanente como la acción de los sismos, siendo esta última la que controla el diseño de los muros.

\footnotetext{
Artículo recibido el 13 de junio de 2002 y aprobado para su publicación el 23 de octubre de 2003. Se aceptarán comentarios y/o discusiones hasta cinco meses después de su publicación.

(1) Departamento de Ingeniería Civil, Universidad de Chile, Casilla 228-3, Santiago, Chile, mastroza@cec.uchile.c1

${ }^{(2)}$ Ingeniero Civil, Universidad de Chile, aschmidt@manquehue.net
} 
Con el propósito de lograr un diseño que garantice un buen comportamiento ante sismos de diferente severidad, y teniendo en cuenta las necesidades que generan los criterios incorporados en las normas de diseño sísmico modernas, donde se reconocen diferentes niveles de desempeño para la estructura (estados límites), es necesario conocer la capacidad de deformación de los muros de albañilería confinada para estos estados. Por ello, el principal objetivo de este trabajo es establecer los niveles de deformación de los muros de albañilería confinada para los estados límites que interesan en su diseño; con este propósito se utiliza la información proporcionada por algunos estudios experimentales realizados los últimos 25 años en Chile (Diez, 1987; Herrera, 1992; Muñoz, 1992), México (Sánchez, 1998; Díaz y Vásquez, 1993; Alcocer et al., 1997; Aguilar, 1997) y Venezuela (Carrillo y Molina, 1997; Castilla, 1998).

\section{ANTECEDENTES}

Los muros de albañilería sin refuerzo presentan importantes limitaciones para resistir acciones sísmicas debido a la baja capacidad de la albañilería para resistir tracciones y a su comportamiento frágil una vez que se produce el agrietamiento. Dadas estas características de la albañilería sin refuerzo, es necesario reforzar los muros y una forma de hacerlo es por medio de elementos esbeltos de hormigón armado (pilares y cadenas) que confinan el paño de albañilería y que deben cumplir con los requisitos establecidos por las normas de diseño y cálculo de estructuras de albañilería (INN, 1997; NTCM-2001, 2001). El confinamiento se logra en la medida que los pilares y cadenas enmarquen completamente el paño de albañilería proporcionándole ante acciones contenidas en el plano del muro capacidad de deformación lateral y de disipación de energía una vez que se agrieta el paño. Para lograr el confinamiento, se deben cumplir con los detalles siguientes (Gallegos, 1987):

(i) El paño de albañilería se debe construir en primer lugar, dejando sus bordes verticales "endentados", para luego colocar el hormigón de los elementos de confinamiento.

(ii) Los elementos de confinamiento no deben interrumpirse.

(iii) Los paños de albañilería deben tener una forma aproximadamente cuadrada, debiéndose colocar pilares de confinamiento intermedios si es necesario.

(iv) Los paños de albañilería no deben presentar fallas de aplastamiento por compresión. Por tal motivo, se recomienda el uso de unidades macizas o perforadas con un porcentaje reducido de perforaciones, menor que un $30 \%$.

El comportamiento de un muro de albañilería confinada depende de la calidad de la albañilería, de las dimensiones y de la cuantía de refuerzo de los elementos de confinamiento, del trabajo conjunto que se logre entre el paño de albañilería y los elementos de confinamiento, de la esbeltez del muro y de la existencia de armadura horizontal en el paño de albañilería. Las armaduras de refuerzo de los elementos de confinamiento deben resistir tanto las tracciones que producen los momentos de flexión debido a las acciones que actúan en el plano del muro como los esfuerzos de flexión y corte que producen las cargas perpendiculares al plano del muro. 
Además, es necesario una mayor concentración de estribos en las zonas por donde se tiende a propagar el agrietamiento del paño, zonas críticas (fig 1), para evitar que la grieta atraviese los pilares o la cadena del muro y se produzca una rotulación en los elementos de confinamiento.

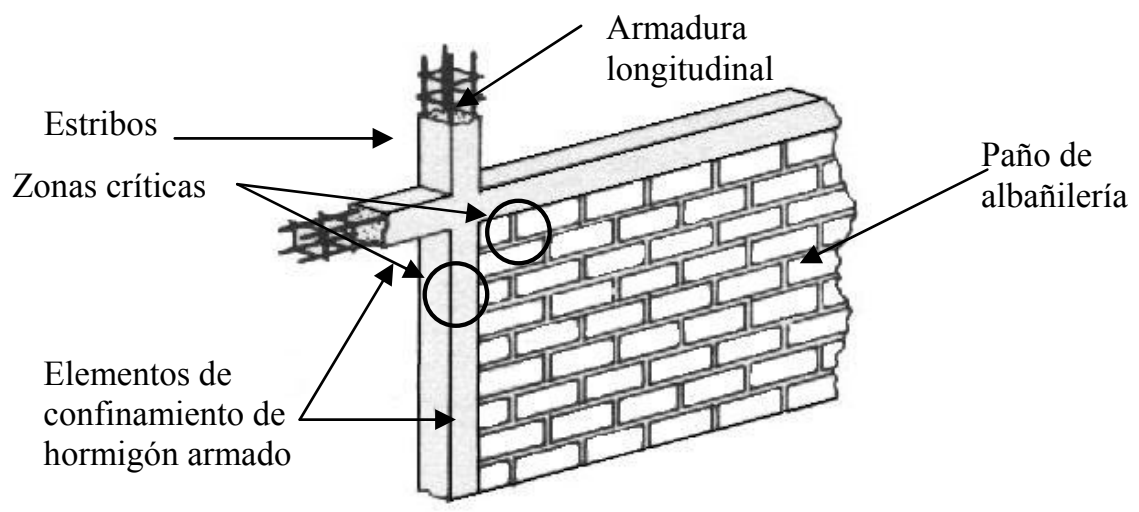

Figura 1. Configuración de un muro de albañilería confinada

Los modos de fallas que pueden presentar este tipo de muros son los siguientes (Gallegos, 1989; Paulay y Priestley, 1991):

i.- Falla de flexión: Este modo de falla se puede presentar en muros esbeltos, cuando se generan tracciones importantes en los pilares de confinamiento, produciéndose la fluencia de las armaduras longitudinales y una falla de compresión por flexión en el talón comprimido del muro.

ii.- Falla de corte por deslizamiento: Este modo de falla se produce por un deslizamiento a lo largo de la junta horizontal de mortero como consecuencia de una falla de adherencia por corte en la junta. Este deslizamiento produce un mecanismo del tipo "columna corta" en los pilares, como se muestra en la fig 2 .

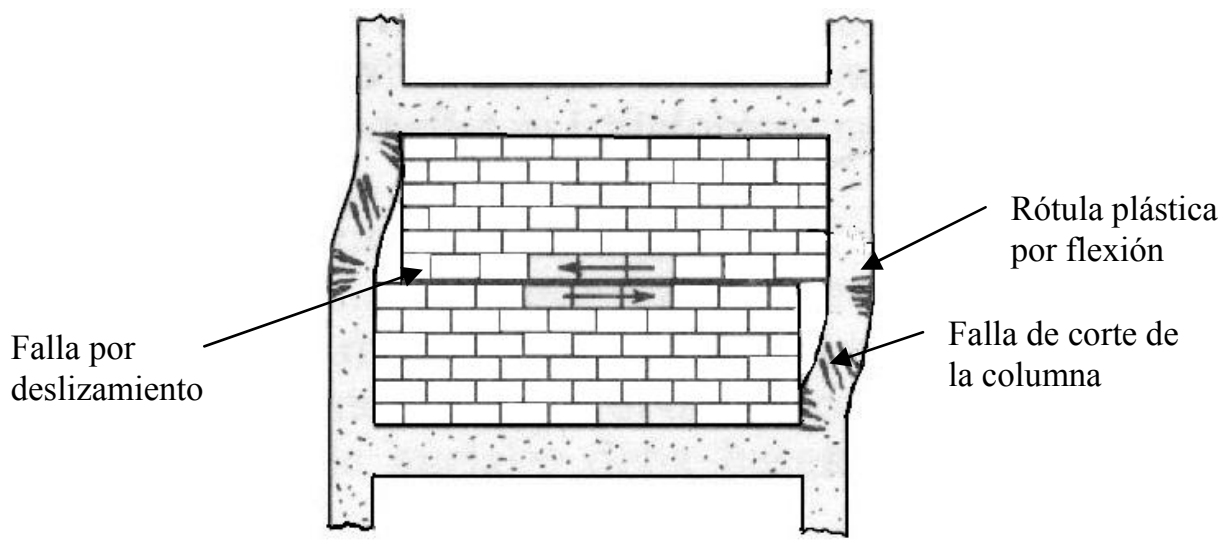

Figura 2. Falla de corte por deslizamiento

iii.- Falla de corte: Esta falla se caracteriza por un agrietamiento diagonal del paño de albañilería como se muestra en la fig 3 y es consecuencia de las tensiones de tracción diagonal que se producen en el paño. Para evitar la propagación de la grieta diagonal en los elementos de 
confinamiento es necesario reforzar las zonas críticas de estos elementos, especialmente cuando la albañilería es de buena calidad y el paño de albañilería es largo.
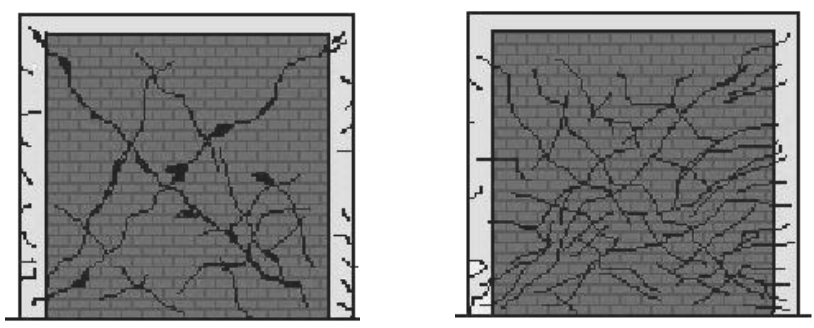

Figura 3. Falla de corte sin y con armadura horizontal en el paño

iv.- Falla de aplastamiento por compresión diagonal: Esta falla es producto del efecto de puntal que se produce cuando se separa el paño de albañilería de los elementos de confinamiento, como se muestra en la fig 4. Esta situación genera grandes tensiones de compresión en las esquinas del muro, las que pueden provocar la falla por aplastamiento de la zona cuando la albañilería es de baja calidad o cuando se usan unidades del tipo rejilla de paredes delgadas.

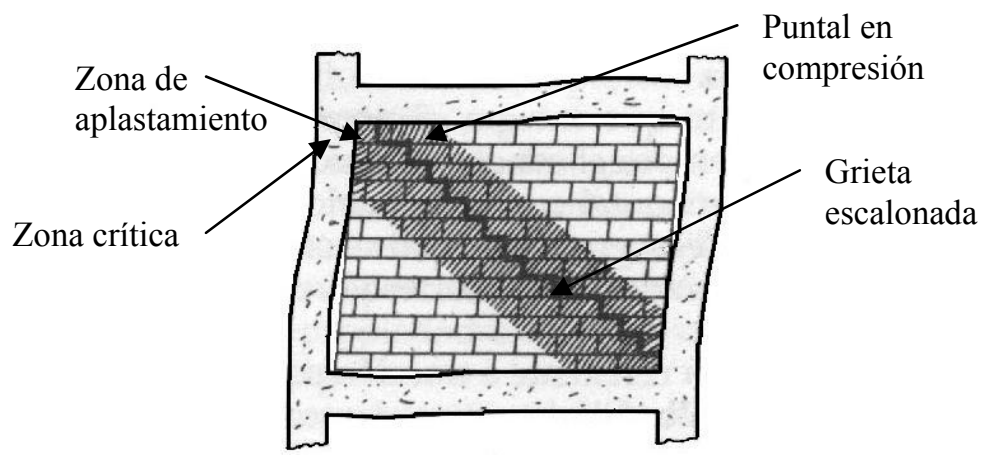

Figura 4. Falla por aplastamiento por compresión diagonal

\section{ESTADOS LÍMITES DE MUROS DE ALBAÑILERÍA CONFINADA}

Los estados límites o los niveles de desempeño reconocidos en el diseño quedan definidos por un patrón de daños, el que depende del nivel de deformación (deriva o distorsión angular) alcanzado por los elementos primarios de una estructura (Bonelli, 1999).

Para los efectos de este trabajo, los estados límites que se reconocen en un muro de albañilería confinada son los siguientes:

i.- Ultimo nominal (ELU): Estado más allá del cual no queda asegurado que no se producirá el colapso. Para efectos prácticos se ha considerado que este estado se alcanza cuando el muro ha experimentado un deterioro del $20 \%$ de la fuerza máxima resistida durante el ensayo. Para este estado, los daños del muro son apreciables, observándose grietas de gran ancho (10 mm o más) y difíciles de reparar. Además de la degradación de la resistencia (20\%) se presenta un importante deterioro de la rigidez. 
ii.- Resistencia (ELR): Estado en el cual el muro alcanza la máxima capacidad de carga. El muro presenta un daño importante pero tiene un margen razonable de seguridad contra el colapso parcial o total.

iii.- Daño controlado(ELDC): Estado en el cual se presenta la formación de un patrón estable de agrietamiento diagonal. El estado del muro permite repararlo en un tiempo razonable y no hay ningún riesgo para las personas y los contenidos.

iv.-Operacional (ELO): Estado en el cual se presenta el inicio del agrietamiento diagonal. El daño del muro es muy limitado, conservando toda su capacidad resistente y parte importante de su rigidez; el riesgo para los habitantes como resultado de este daño es nulo.

v.- Servicio (ELS): Estado en el cual se presenta el inicio de agrietamiento visible del muro y corresponde al nivel donde se produce el término del rango elástico de respuesta del muro.

A modo de ejemplo, en la fig 5 se destaca cada uno de estos estados límites en las curvas de histéresis de un muro de albañilería confinada construido con unidades cerámicas hechas a máquina.

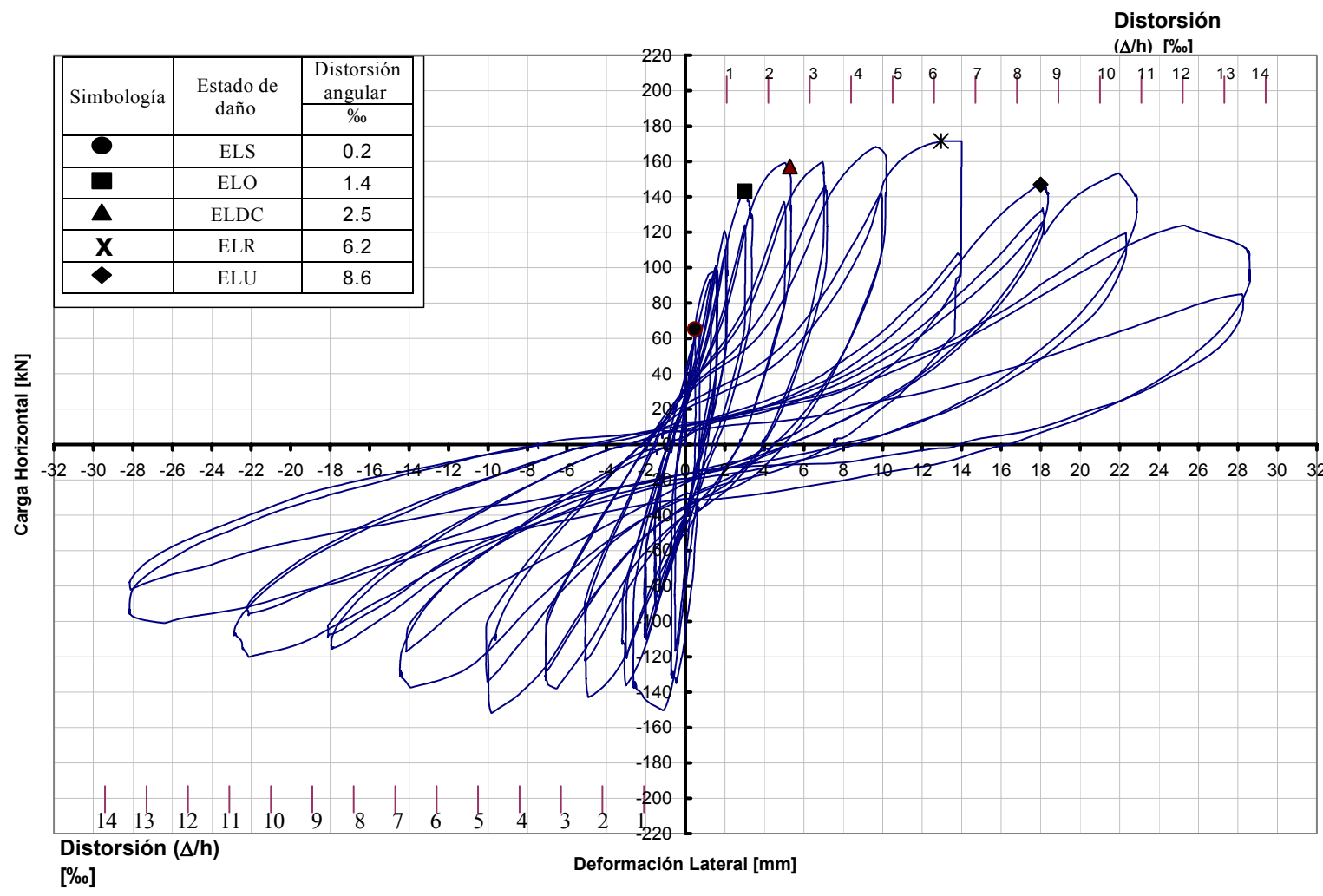

Figura 5. Identificación de los estados límites en las curvas de histéresis de un muro de albañilería confinada

En la fig 6 se muestra el estado de daño para cuatro de estos estados límite. 


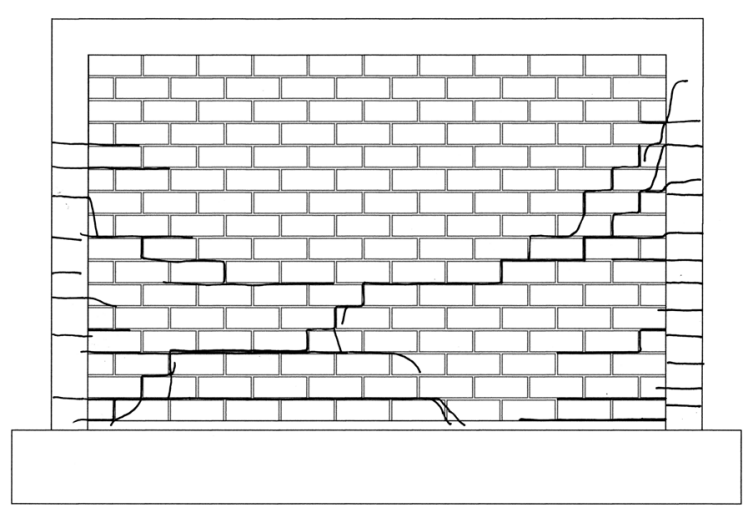

ELS

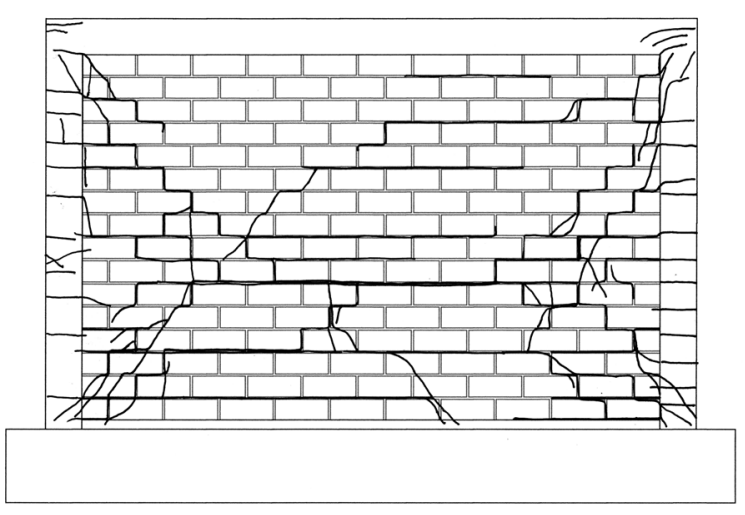

ELDC

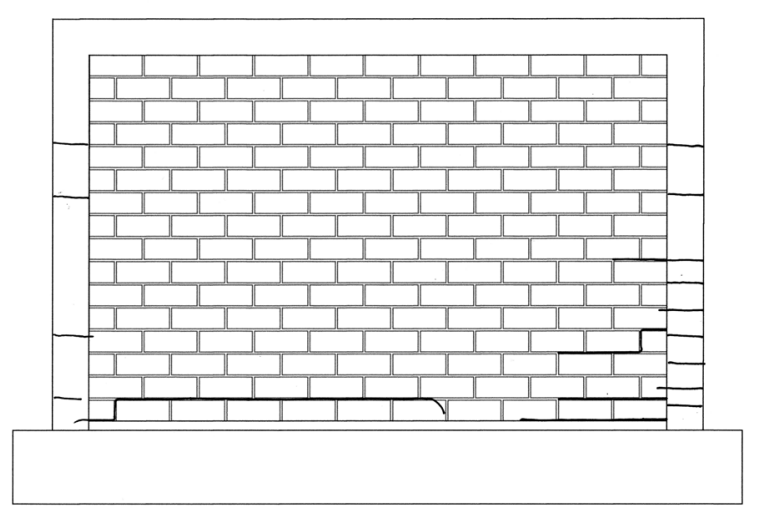

ELO

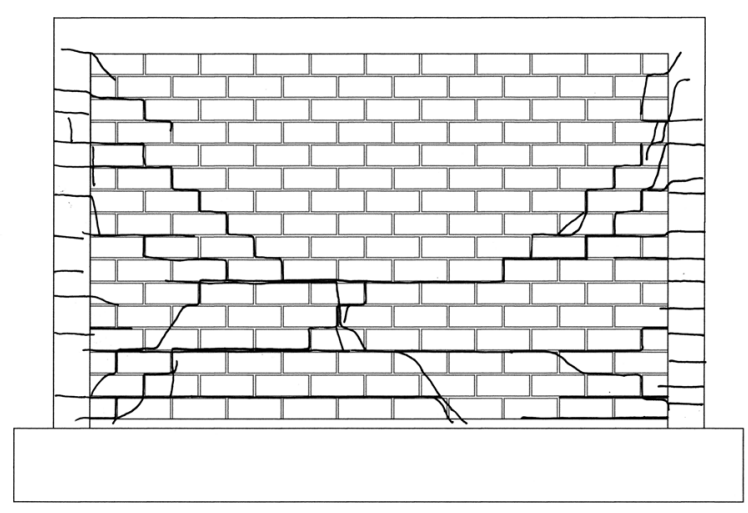

ELU

Figura 6. Estados de daños para los estados límites ELS, ELO, ELDC y ELU

\section{CAPACIDAD DE DEFORMACIÓN DE MUROS DE ALBAÑILERÍA CONFINADA}

Para determinar la capacidad de deformación correspondiente a cada uno de los cinco estados límites destacados, se reunió una base de datos de 52 muros de albañilería confinada sometidos a cargas laterales alternadas cuyo comportamiento estuvo controlado por la falla de corte. Esta base de datos corresponde a los ensayos de muros de albañilería confinada realizados en tres países latinoamericanos: Chile, México y Venezuela. La información reunida para cada muro corresponde a: propiedades geométricas, cuantía del refuerzo horizontal del paño de albañilería, cuantía del refuerzo longitudinal de los pilares y cadenas, cuantía de estribos de los pilares y las cadenas, propiedades mecánica de los materiales del muro, tipo de probeta ensayadas en los estudios revisados (fig 7), carga vertical aplicada sobre los muros y niveles de carga y deformación para los distintos estados límites considerados. Detalles de esta base de datos se entregan en la memoria de título de Andrés Schmidt (Schmidt, 2002). 


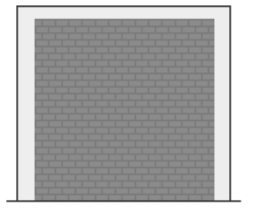

a)
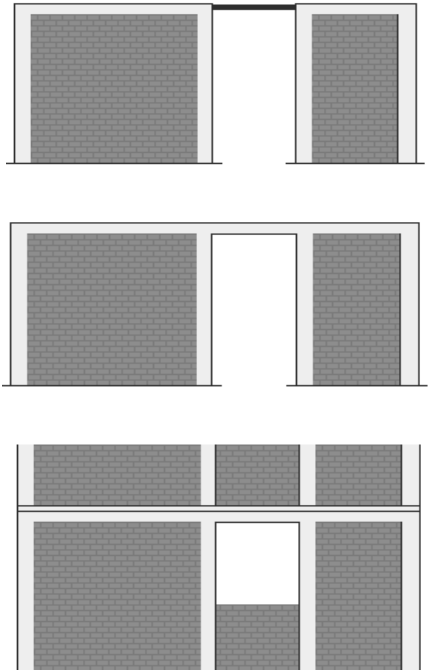

b)

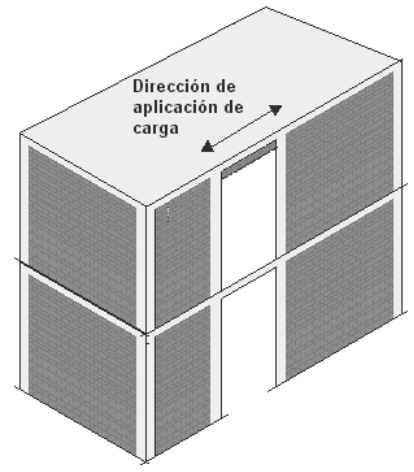

c)

Figura 7. a) Muros simples con esbelteces variables b) Muros dobles con distintos grados de acoplamiento c) Sistema tridimensional construido con muros acoplados y muros simples

Los datos de los muros utilizados en este trabajo se detallan en la tabla 1. En esta tabla el tipo de unidad se identifica de la forma siguiente: ladrillo hecho a máquina, C. Máquina, ladrillo hecho a mano, C. Mano, y bloque de hormigón, B. Hormigón. La distorsión angular indicada en esta tabla se obtuvo dividiendo el desplazamiento horizontal relativo entre el extremo superior e inferior del muro por su altura, expresando esta relación en porcentaje. Por otra parte, la esbeltez corresponde a la relación entre el alto y el largo del muro.

De la tabla 1 se aprecia que la mayoría de los muros tienen esbelteces del orden de 1.0, como se aprecia en la fig 8.

Esbeltez que pres entan los muros revisados de los estudios experimentales

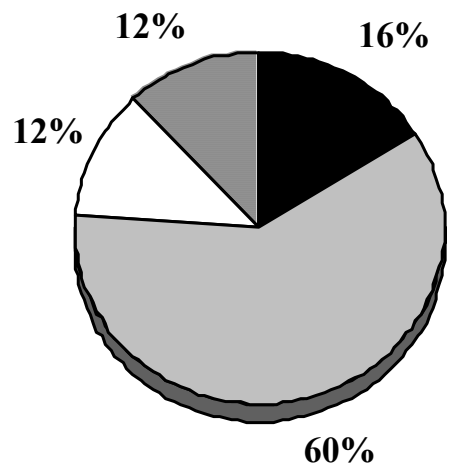

口 $1.0<$ Es beltez $\leq 2.0$

$\square 0.75<$ Es beltez $\leq 1.0$

Es beltez $\leq 0.75$

Muros 3D Muros

Acoplados

Figura 8. Distribución porcentual de la base de datos según la esbeltez de los muros 
Maximiliano Astroza y Andrés Schmidt

Tabla 1. Distorsiones asociadas con los estados límites considerados para los muros de albañilería confinada

\begin{tabular}{|c|c|c|c|c|c|c|c|c|c|c|c|c|c|c|c|c|c|c|}
\hline \multicolumn{2}{|c|}{ MUROS SIMPLES } & & \multicolumn{5}{|c|}{ DISTORSIONES POSITIVAS [\%] } & \multicolumn{5}{|c|}{ DISTORSIONES NEGATIVAS [\%] } \\
\hline Ficha & Autor & Denom & Tipo unidad & $\begin{array}{c}\tau \mathrm{m} \\
\mathrm{kg} / \mathrm{cm}^{2}\end{array}$ & $\begin{array}{c}\mathrm{f}^{\prime} \mathrm{m} \\
\mathrm{kg} / \mathrm{cm}^{2}\end{array}$ & Esbeltez & $\begin{array}{c}\text { Arm Horiz } \\
\% \\
\end{array}$ & $\begin{array}{c}\text { Tension vert } \\
\mathrm{kg} / \mathrm{cm}^{2}\end{array}$ & ELS & ELO & ELDC & ELR & ELU & ELS & ELO & ELDC & ELR & ELU \\
\hline 1 & Hererra & A11 & C. Maquina & 8 & 122 & 1 & 0 & 0.00 & 0.09 & 0.15 & 0.21 & 0.21 & 0.30 & 0.10 & 0.24 & 0.36 & 0.36 & \\
\hline 2 & Hererra & A12 & C. Maquina & 8 & 122 & 1 & 0 & 2.38 & 0.35 & 0.35 & 0.35 & 0.35 & & 0.33 & 0.42 & 0.42 & 0.42 & \\
\hline 3 & Hererra & A13 & C. Maquina & 8 & 122 & 1 & 0 & 4.76 & 0.18 & 0.18 & 0.18 & 0.18 & & 0.19 & 0.19 & 0.19 & 0.19 & \\
\hline 4 & Hererra & Al4 & C. Maquina & 8 & 122 & 1 & 0 & 4.76 & 0.05 & 0.11 & 0.21 & 0.21 & & 0.06 & 0.10 & 0.16 & 0.16 & \\
\hline 5 & Hererra & $\mathrm{A} 2$ & C. Maquina & 8 & 122 & 1 & 0 & 2.38 & 0.16 & 0.16 & 0.16 & 0.16 & & 0.13 & 0.13 & 0.13 & 0.13 & \\
\hline 6 & Hererra & B11 & C. Mano & 2.35 & S.I. & 1 & 0 & 0.00 & 0.06 & 0.06 & 0.10 & 0.10 & & 0.07 & 0.07 & 0.26 & 0.26 & \\
\hline 7 & Hererra & B12 & C. Mano & 2.35 & S.I. & 1 & 0 & 4.76 & 0.15 & 0.15 & 0.28 & 0.28 & & 0.24 & 0.24 & 0.36 & 0.36 & \\
\hline 8 & Hererra & B13 & C. Mano & 2.35 & S.I. & 1 & 0 & 4.76 & 0.12 & 0.12 & 0.18 & 0.18 & & 0.13 & 0.13 & 0.19 & 0.19 & \\
\hline 9 & Hererra & B14 & C. Mano & 2.35 & S.I. & 1 & 0 & 4.76 & 0.11 & 0.11 & 0.22 & 0.46 & & 0.11 & 0.11 & 0.20 & 0.50 & \\
\hline 10 & Hererra & $\mathrm{B} 2$ & C. Mano & 2.35 & S.I. & 1 & 0 & 2.38 & 0.24 & 0.24 & 0.46 & 0.46 & & 0.24 & 0.24 & 0.62 & 0.62 & \\
\hline 11 & Diez & MRGl & C. Maquina & 10.56 & 137 & 1 & 0 & 0.00 & 0.04 & 0.14 & 0.26 & 0.26 & 0.58 & 0.05 & 0.11 & 0.32 & 0.32 & 0.73 \\
\hline 12 & Diez & MRG2 & C. Maquina & 10.56 & 137 & 1 & 0 & 0.00 & 0.03 & 0.14 & 0.29 & 0.73 & 1.00 & 0.01 & 0.08 & 0.17 & 0.29 & 0.58 \\
\hline 13 & Diez & MRE1 & C. Maquina & 10.56 & 137 & 2 & 0 & 0.00 & 0.09 & 0.20 & 0.30 & 1.30 & 2.46 & 0.03 & 0.20 & 0.32 & 1.17 & 1.60 \\
\hline 14 & Diez & MRE2 & C. Maquina & 10.56 & 137 & 2 & 0 & 0.00 & 0.07 & 0.14 & 0.22 & 0.99 & 1.38 & 0.10 & 0.20 & 0.24 & 0.96 & 1.33 \\
\hline 15 & Muñoz & $\mathrm{C} 11$ & B. Hormigon & 7.1 & 91.7 & 1 & 0 & 4.17 & 0.14 & 0.26 & 0.26 & 0.51 & & 0.23 & & 0.27 & 0.50 & \\
\hline 16 & Muñoz & $\mathrm{Cl} 2$ & B. Hormigon & 7.07 & 124.1 & 1 & 0 & 4.17 & 0.13 & & 0.21 & 0.39 & & 0.23 & & 0.29 & 0.61 & \\
\hline 17 & Muñoz & $\mathrm{Cl} 3$ & B. Hormigon & 7.1 & 152.3 & 1 & 0 & 4.17 & 0.14 & 0.13 & 0.26 & 0.38 & & 0.14 & & 0.16 & 0.43 & \\
\hline 18 & Muñoz & $\mathrm{C} 14$ & B. Hormigon & 7.1 & 152.3 & 1 & 0 & 0.00 & 0.12 & 0.13 & 0.21 & 0.62 & & 0.12 & 0.13 & 0.22 & 0.50 & \\
\hline 19 & Alcocer & N1 & C. Maquina & 16.4 & 149 & 1 & 0 & 4.00 & 0.10 & 0.10 & 0.12 & 0.24 & & 0.05 & 0.10 & & 0.23 & \\
\hline 20 & Alcocer & N2 & C. Maquina & 16.1 & 172 & 1 & 0.05 & 4.00 & 0.10 & 0.10 & 0.15 & 0.40 & & 0.10 & 0.10 & 0.15 & 0.40 & \\
\hline 21 & Alcocer & N3 & C. Maquina & 12.1 & 162 & 1 & 0.05 & 4.00 & 0.10 & 0.10 & 0.40 & 0.50 & 0.60 & 0.10 & 0.10 & & 0.40 & 0.50 \\
\hline 22 & Alcocer & $\mathrm{N} 4$ & C. Maquina & 7.5 & 158 & 1 & 0.19 & 4.00 & 0.10 & 0.10 & 0.13 & 0.60 & 0.72 & 0.10 & 0.10 & 0.11 & 0.60 & \\
\hline 23 & Aguilar & Ml & C. Mano & 5.7 & 49 & 1 & 0.211 & 5.00 & 0.22 & 0.22 & 0.22 & 0.57 & 1.04 & 0.18 & 0.18 & & 0.57 & \\
\hline 24 & Aguilar & $\mathrm{M} 2$ & C. Mano & 3.9 & 37 & 1 & 0 & 5.00 & 0.13 & 0.13 & 0.44 & 0.44 & 0.72 & 0.10 & 0.10 & & 0.42 & \\
\hline 25 & Aguilar & $\mathrm{MB}$ & C. Mano & 3.8 & 31 & 1 & 0.071 & 5.00 & 0.17 & 0.17 & & 0.61 & 1.34 & 0.17 & & 0.48 & 0.56 & \\
\hline 26 & Aguilar & M4 & C. Mano & 4 & 38 & 1 & 0.19 & 5.00 & 0.05 & 0.12 & & 0.83 & 1.79 & 0.17 & 0.17 & & 0.59 & \\
\hline 27 & Carrillo/Molina & Murol & C. Mano & 6.61 & 92.78 & 0.71 & 0 & 0.00 & 0.07 & & & 0.08 & 0.45 & 0.08 & & & 0.08 & 0.16 \\
\hline 28 & Carrillo/Molina & Muro2 & C. Mano & 6.61 & 92.78 & 0.71 & 0 & 9.28 & 0.05 & & & 0.28 & & 0.05 & & & 0.29 & \\
\hline 29 & Carrillo/Molina & Muro3 & C. Mano & 6.61 & 92.78 & 0.71 & 0 & 4.64 & 0.07 & & & 0.09 & 0.22 & 0.08 & & & 0.11 & \\
\hline 30 & Carrillo/Molina & Muro4 & C. Mano & 6.61 & 92.78 & 1.13 & 0 & 6.96 & 0.07 & & & 0.46 & & 0.07 & & & 0.13 & \\
\hline 31 & Carrillo/Molina & Muro5 & C. Mano & 6.61 & 92.78 & 1.13 & 0 & 0.00 & 0.07 & & & 0.73 & 0.87 & 0.07 & & & 0.73 & \\
\hline 32 & Carrillo/Molina & Muro6 & C. Mano & 6.61 & 92.78 & 1.13 & 0 & 13.92 & 0.07 & & & 0.15 & 0.29 & 0.04 & & & 0.29 & \\
\hline 33 & Carrillo/Molina & Muro7 & C. Mano & 6.61 & 92.78 & 0.95 & 0 & 13.92 & 0.07 & & & 0.36 & 0.67 & 0.07 & & & 0.44 & 0.66 \\
\hline 34 & Carrillo/Molina & Muro8 & C. Mano & 6.61 & 92.78 & 0.95 & 0 & 0.00 & 0.07 & & & 0.14 & & 0.07 & & & 0.22 & \\
\hline 35 & Carrillo/Molina & Muro9 & C. Mano & 6.61 & 92.78 & 0.95 & 0 & 4.64 & 0.06 & & & 0.87 & & 0.15 & & & 0.65 & 0.86 \\
\hline 36 & Carrillo/Molina & Murol0 & C. Mano & 6.61 & 92.78 & 0.95 & 0 & 0.00 & 0.05 & & & 0.56 & 1.28 & 0.17 & & & 0.73 & 1.53 \\
\hline 37 & Castilla & Muro 1 & B. Hormigon & 7.64 & 77.83 & 0.97 & 0 & 7.78 & 0.16 & & & 0.39 & 0.45 & 0.05 & & & 0.46 & \\
\hline 38 & Castilla & Muro 2 & B. Hormigon & 7.64 & 77.83 & 0.97 & 0 & 3.89 & 0.10 & & & 0.38 & & 0.04 & & & 0.43 & \\
\hline 39 & Castilla & Muro 3 & B. Hormigon & 7.64 & 77.83 & 0.97 & 0 & 0.00 & 0.13 & & & 0.68 & & 0.04 & & & 0.67 & \\
\hline 40 & Castilla & Muro 4 & B. Hormigon & 7.64 & 77.83 & 0.74 & 0 & 5.84 & 0.06 & & & 0.31 & & 0.06 & & & 0.31 & \\
\hline 41 & Castilla & Muro 5 & B. Hormigon & 7.64 & 77.83 & 0.74 & 0 & 0.00 & 0.04 & & & 0.17 & 0.31 & 0.04 & & & 0.21 & \\
\hline 42 & Castilla & Muro 6 & B. Hormigon & 7.64 & 77.83 & 0.74 & 0 & 3.89 & 0.02 & & & 0.25 & & 0.02 & & & 0.35 & \\
\hline 43 & Castilla & Muro 7 & B. Hormigon & 7.64 & 77.83 & 1.26 & 0 & 7.78 & 0.13 & & & 0.67 & 0.77 & 0.25 & & & 0.70 & \\
\hline 44 & Castilla & Muro 8 & B. Hormigon & 7.64 & 77.83 & 1.26 & 0 & 0.00 & 0.03 & & & 0.83 & & 0.06 & & & 0.83 & \\
\hline 45 & Castilla & Muro 9 & B. Hormigon & 7.64 & 77.83 & 1.26 & 0 & 3.89 & 0.03 & & & 0.75 & & 0.08 & & & 0.71 & \\
\hline
\end{tabular}

\section{MUROS ACOPLADOS (DOBLES)}

\begin{tabular}{|c|c|c|c|c|c|c|c|c|c|c|c|c|c|c|c|c|c|c|}
\hline 46 & Sanchez & WBW & C. Mano & 10 & 53 & - & 0 & 5 & 0.12 & 0.12 & 0.60 & 0.60 & & 0.12 & 0.12 & 0.34 & 0.15 & \\
\hline 47 & Sanchez & W-W & C. Mano & 10 & 48 & - & 0 & 5 & 0.10 & 0.10 & 0.60 & 0.60 & 1.00 & 0.12 & 0.12 & 0.54 & 0.54 & 0.80 \\
\hline 48 & Sanchez & www & C. Mano & 10 & 53 & - & 0 & 5 & 0.18 & 0.18 & 0.74 & 0.74 & & 0.14 & 0.14 & 0.60 & 0.60 & \\
\hline 49 & Sanchez et al & WBW-B & C. Mano & 8 & 45 & - & 0.091 & 5 & 0.18 & 0.18 & 0.74 & 0.74 & 0.79 & 0.14 & 0.14 & 0.60 & 0.60 & \\
\hline 50 & Sanchez et al & WBW-E & C. Mano & 7 & 70 & - & 0.091 & 5 & 0.13 & 0.13 & 0.45 & 0.22 & 0.56 & 0.18 & 0.18 & 0.50 & 0.50 & \\
\hline
\end{tabular}

MURO TRIDIMENSIONAL

\begin{tabular}{|c|l|l|l|l|l|l|l|l|l|l|l|l|l|l|l|l|l|l|l|}
\hline 51 & Sanchez et al & nivel1 & C. Mano & 6 & 54 & - & 0 & 5 & 0.04 & 0.12 & 0.21 & 0.20 & 0.50 & 0.04 & 0.07 & 0.20 & 0.39 & 0.54 \\
52 & Sanchez et al & nivel2 & C. Mano & 6 & 54 & - & 0 & 5 & 0.02 & 0.13 & & 0.23 & & 0.02 & 0.08 & & 0.21 & \\
\hline
\end{tabular}

S.I. : Sin Información

Además, la mayor cantidad de datos corresponde a muros construidos con unidades del tipo cerámico, como se muestra en la fig 9. 


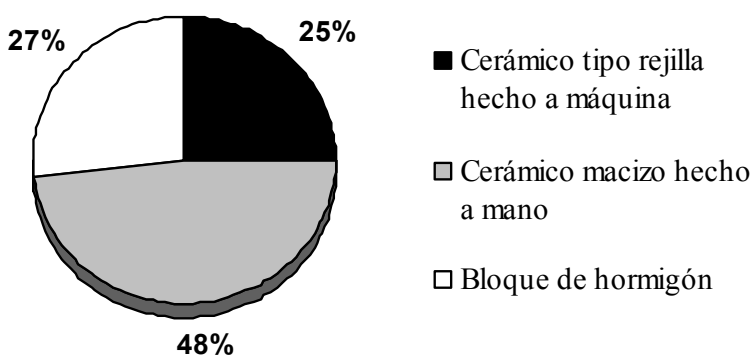

Figura 9. Distribución porcentual de la base de datos según el tipo de unidad de albañilería de los muros

Las propiedades mecánicas de las albañilerías se indican en la tabla 1 y varían en los intervalos indicados en la tabla 2.

Tabla 2. Propiedades mecánicas de las albañilerías

\begin{tabular}{|l|c|c|}
\hline \multicolumn{1}{|c|}{ Unidad de albañilería } & $\mathbf{f}^{\prime} \mathbf{m}$, en $\mathbf{M P a}$ & $\tau_{\mathbf{m}}$, en MPa \\
\hline Cerámico hecho a máquina & $12-17$ & $0.98-1.64$ \\
\hline Cerámico hecho a mano & $2-9$ & $0.2-1.0$ \\
\hline Bloques de hormigón & $5-15$ & $0.7-1.0$ \\
\hline
\end{tabular}

La determinación de la capacidad de deformación para cada uno de los estados límites se hizo de un análisis estadístico de los valores experimentales de las distorsiones angulares asociadas a cada estado. Del análisis estadístico del conjunto de datos, se obtuvo la distribución estadística que mejor se ajusta a la distribución de frecuencias relativas acumuladas de las distorsiones angulares de los muros, a través de una prueba de bondad de ajuste de KolmogorovSmirnov y la prueba de Chi cuadrado $\left(\chi^{2}\right)$. Las distorsiones angulares se determinan aceptando que el porcentaje de muros de la muestra (fracción defectuosa) que alcanzan el estado límite considerado a una distorsión angular menor que el valor establecido, corresponde al 10, 20 y 50\% de los muros.

El desarrollo de este análisis estadístico se realizó en dos etapas (Schmidt, 2002), en la primera se consideran todos los muros sin hacer distinción de ningún tipo y en la segunda se agrupan los muros de acuerdo con el tipo de unidad y con la magnitud de la carga vertical. En este último caso se consideró un intervalo de baja de tensión de compresión (menores que 0.4 $\mathrm{MPa}$ ) y uno de alta tensión (mayores o iguales a $0.4 \mathrm{MPa}$ ). En ambas etapas, las distorsiones de los ciclos positivos y negativos se consideran como datos independientes para cada estado límite y los muros de esbeltez 0.97 y 0.95 como muros de esbeltez uno. Los muros con refuerzo horizontal en su interior sólo se consideraron en la segunda etapa, para los estados límites de servicio y operacional, aceptando que la presencia de este tipo de refuerzo no afecta a estos dos estados (Alcocer et al., 19997; Gallegos, 1989).

La base de datos de la tabla 1 permite analizar estadísticamente los efectos del tipo de unidad y de la carga vertical en la capacidad de deformación sólo en algunos estados límites. En cambio, el efecto de la esbeltez del muro y de la presencia de armadura horizontal de refuerzo no es posible analizarlo. 
Cuando se consideran los datos de la tabla 1 como un todo, sin hacer distinción de los distintas variables que están involucradas en los estudios experimentales revisados, se obtienen las distribuciones estadísticas de la distorsión angular mostradas en la fig 10 para cada estado límite. Los valores de las distorsiones angulares que resultan de estas distribuciones se entregan en la tabla 3 para tres fracciones defectuosas.
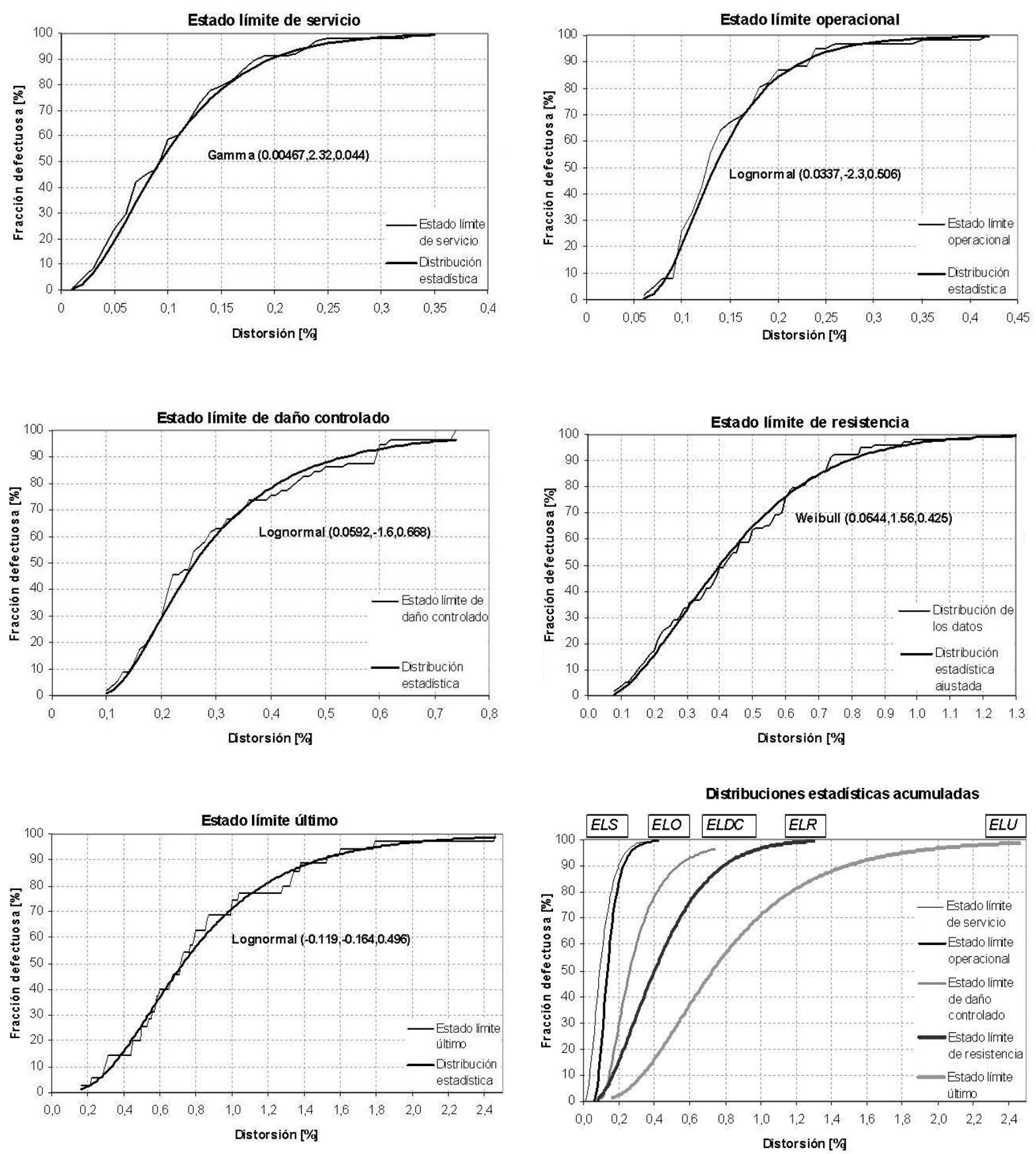

Figura 10. Distribuciones estadísticas de las distorsiones angulares para los estados límites de diseño 
Tabla 3. Distorsiones angulares considerando los muros como un todo

\begin{tabular}{|l|c|c|c|}
\hline \multirow{2}{*}{ ESTADO LÍMITE } & \multicolumn{3}{|c|}{ DISTORSIÓN [\%] } \\
\cline { 2 - 4 } & $\mathbf{3 0} \%$ & $\mathbf{2 0} \%$ & $\mathbf{5 0} \%$ \\
\cline { 2 - 4 } & 0.04 & 0.05 & 0.09 \\
\hline Servicio (ELS) & 0.09 & 0.10 & 0.13 \\
\hline Operacional (ELO) & 0.14 & 0.17 & 0.26 \\
\hline Daño Controlado (ELDC) & 0.16 & 0.22 & 0.40 \\
\hline Resistencia (ELR) & 0.33 & 0.44 & 0.73 \\
\hline Último (ELU) & \multicolumn{3}{|c|}{} \\
\hline
\end{tabular}

Para efectos prácticos, es recomendable utilizar los valores de la distorsión angular correspondientes a una fracción defectuosa del $20 \%$. La elección de este valor se justifica ya que la distorsión que resulta para cualquier estado límite corresponde a un estado límite superior en menos del 10\% de los casos, como se muestra en la fig 11. Si se aceptan fracciones defectuosas mayores al $20 \%$, el porcentaje de casos que presentan un estado límite superior es mayor que el $10 \%$, provocando una incertidumbre en el desempeño real que pueda presentar la estructura frente a un evento sísmico.

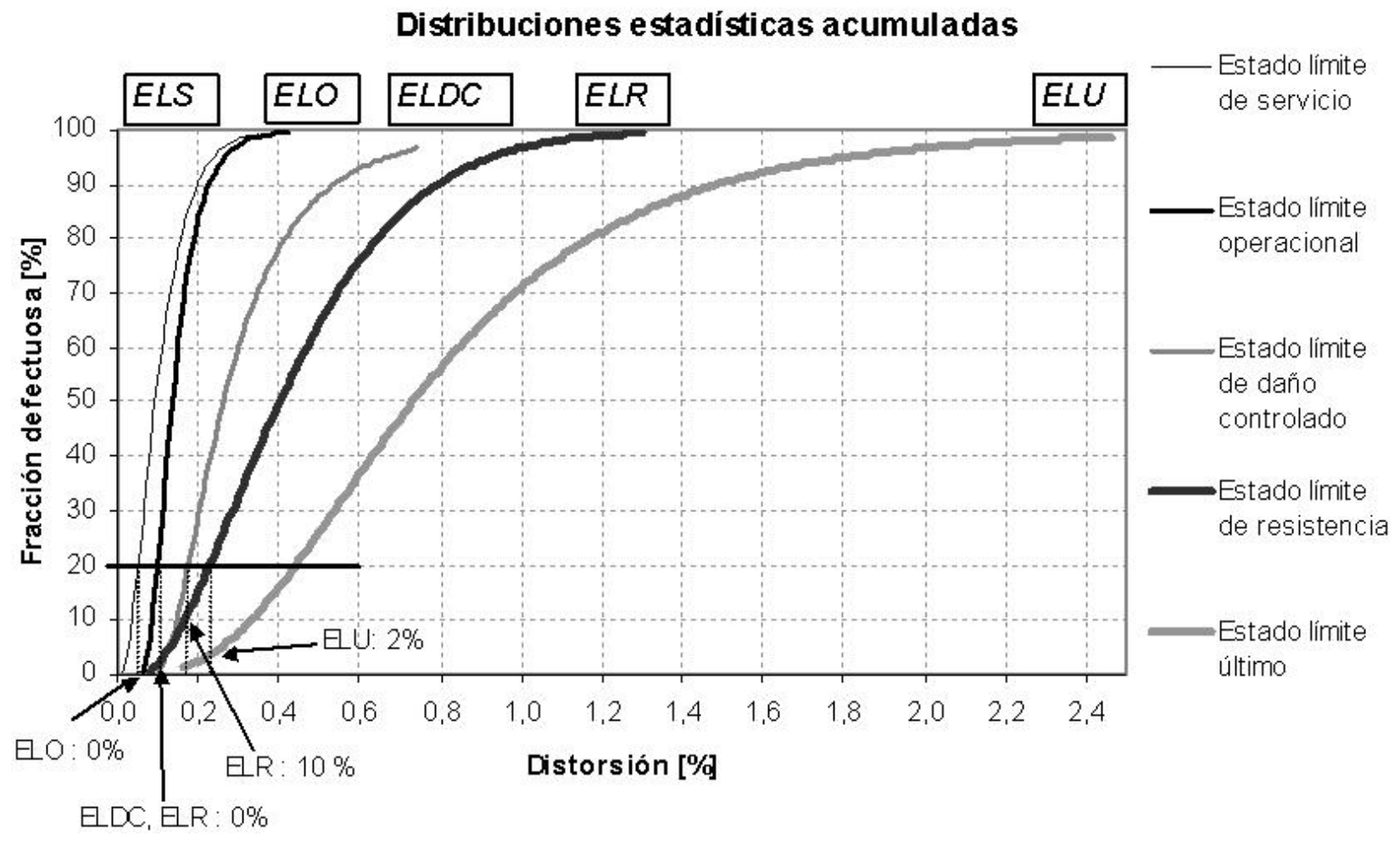

Figura 11. Fracciones defectuosas para los distintos estados límites utilizando los valores de la distorsión angular para un $20 \%$

En relación con los efectos del tipo de unidad, la información disponible sólo permitió hacer un análisis estadístico para los estados límites de servicio y de resistencia, resultando las 
distribuciones estadísticas de la fig 12. En la tabla 4 se entregan los valores de las distorsiones angulares que se obtienen de estas distribuciones estadísticas para cada una de las fracciones defectuosas destacadas.

Tabla 4. Distorsiones angulares considerando el efecto del tipo de unidad

\begin{tabular}{|c|c|c|c|c|c|c|c|c|c|}
\hline \multirow{4}{*}{$\begin{array}{c}\text { ESTADO LÍMITE DE } \\
\text { DISEÑO }\end{array}$} & \multicolumn{9}{|c|}{ DISTORSIÓN (\%) } \\
\hline & \multicolumn{3}{|c|}{$\begin{array}{l}\begin{array}{c}\text { Ladrillo cerámico } \\
\text { hecho a máquina }\end{array} \\
\end{array}$} & \multicolumn{3}{|c|}{$\begin{array}{c}\text { Ladrillo cerámico } \\
\text { hecho a mano }\end{array}$} & \multicolumn{3}{|c|}{ Bloque de hormigón } \\
\hline & \multicolumn{3}{|c|}{ Fracción defectuosa } & \multicolumn{3}{|c|}{ Fracción defectuosa } & \multicolumn{3}{|c|}{ Fracción defectuosa } \\
\hline & $10 \%$ & $20 \%$ & $50 \%$ & $10 \%$ & $20 \%$ & $50 \%$ & $10 \%$ & $20 \%$ & $\mathbf{5 0 \%}$ \\
\hline Estado límite de servicio & 0.03 & 0.05 & 0.09 & 0.06 & 0.07 & 0.11 & 0.05 & 0.08 & 0.12 \\
\hline $\begin{array}{lll}\text { Estado límite } & \text { de } \\
\text { resistencia } & & \end{array}$ & 0.15 & 0.17 & 0.24 & 0.17 & 0.24 & 0.40 & 0.37 & 0.41 & 0.49 \\
\hline
\end{tabular}

Estado límite de servicio

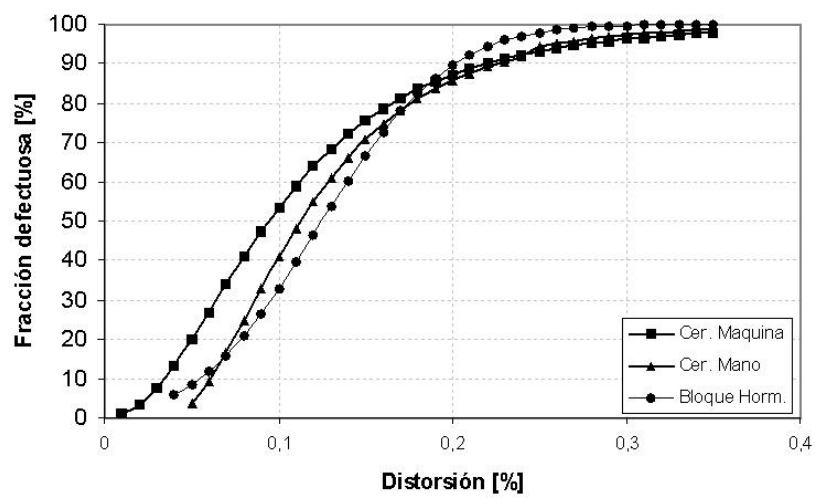

Estado límite de resistencia

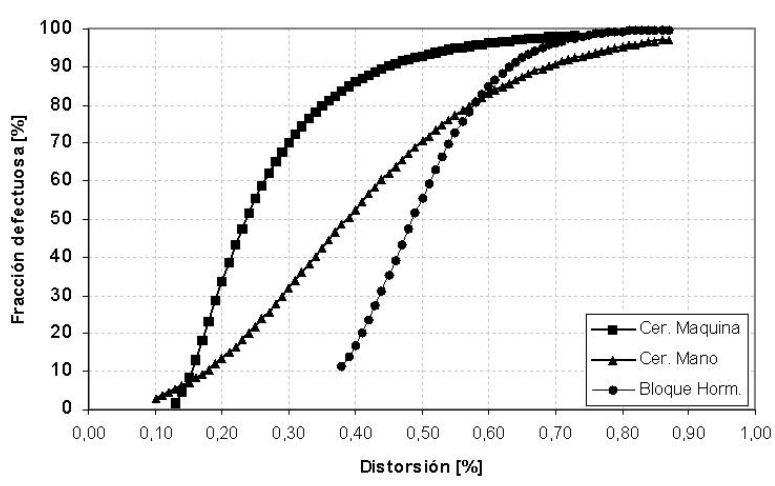

Figura12. Distribuciones estadísticas de las distorsiones angulares según el tipo de unidad

Tabla 5. Distorsiones angulares considerando el efecto de la carga vertical para muros construidos con unidades cerámicas hechas a máquina

\begin{tabular}{|c|c|c|c|c|c|c|}
\hline \multirow{4}{*}{ ESTADO LÍMITE DE DISEÑO } & \multicolumn{6}{|c|}{ DISTORSIÓN (\%) } \\
\hline & \multirow{2}{*}{\multicolumn{3}{|c|}{$\begin{array}{l}\text { Baja carga vertical } \\
\text { Fracción defectuosa }\end{array}$}} & \multirow{2}{*}{\multicolumn{3}{|c|}{$\begin{array}{l}\text { Alta carga vertical } \\
\text { Fracción defectuosa }\end{array}$}} \\
\hline & & & & & & \\
\hline & $10 \%$ & $20 \%$ & $50 \%$ & $10 \%$ & $20 \%$ & $50 \%$ \\
\hline Estado límite de servicio & 0.02 & 0.04 & 0.08 & 0.06 & 0.07 & 0.09 \\
\hline
\end{tabular}

Teniendo en cuenta la información disponible, el efecto de la carga vertical se analiza considerando dos intervalos de tensiones normales debido a esta carga. El primer intervalo corresponde a tensiones menores que $0.4 \mathrm{MPa}$ (Carga vertical baja) y el otro a tensiones mayores o iguales a $0.4 \mathrm{MPa}$ (Carga vertical alta). Este último intervalo es representativo de la situación en que se encuentran los muros de los edificios de albañilería de tres o más pisos. La información disponible permitió hacer un análisis estadístico sólo para el estado límite de servicio para los muros construidos con unidades del tipo cerámico hechas a máquina, resultando las distribuciones estadísticas de la fig 13. En la tabla 5 se indican los valores de las distorsiones angulares, comprobándose que la capacidad de deformación aumenta con la presencia de carga 
vertical para el estado límite de servicio y este aumento es significativo cuando las cargas verticales actuantes producen tensiones normales mayores que $0.4 \mathrm{MPa}$.

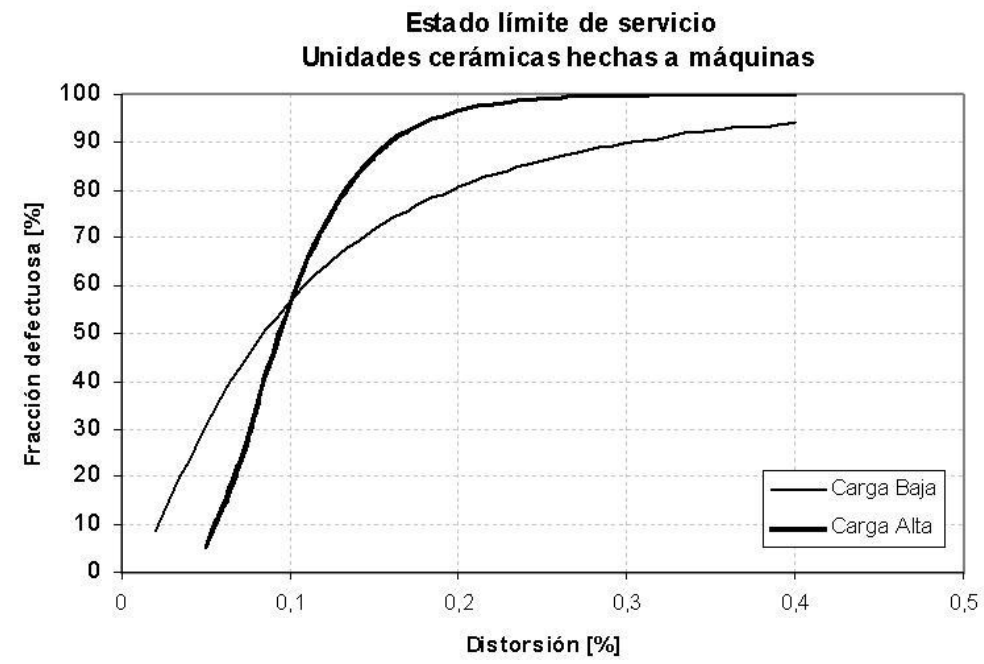

Figura 13. Distribuciones estadísticas de las distorsiones angulares para el Estado Límite de Servicio. Efecto de la carga vertical

\section{ANÁLISIS DE RESULTADOS}

Desde el punto de vista del diseño, la elección de un estado límite depende de varios factores, entre ellos se pueden destacar: consideraciones económicas, interrupción del funcionamiento y protección de los contenidos. Al revisar la información que se encuentra en la literatura relacionada con estos estados, se presentan diferencias en la descripción de los patrones de daño y en los niveles de deformación asociados con cada estado límite.

Para los efectos del cálculo sísmico, las normas reconocen algunos estados límites destacando los valores de los niveles de deformación sin hacer más distinción que la correspondiente a la modalidad de refuerzo utilizada. En particular, la propuesta de norma chilena de diseño sísmico de edificios (Bonelli, 1999), reconoce cuatro estados límites y asigna las distorsiones indicadas en la tabla 6 sin hacer distinción entre los distintos tipos de muros de albañilería reforzada.

Si se compara la descripción de los daños asociados con los estados límites de este trabajo con lo indicado en la tabla 6 , se puede aceptar la equivalencia entre estados destacada en la tabla 7.

Teniendo en cuenta la equivalencia de la tabla 7, se aprecia que las distorsiones de la propuesta de norma chilena de diseño sísmico de edificio son muy altas para muros de albañilería confinada. Esta situación se produce porque las distorsiones angulares propuestas por Bonelli corresponden a valores obtenidos de los ensayos de marcos de hormigón armado rellenos con un muro de albañilería (Decanini et al., 1993), donde el marco de hormigón armado juega un rol 
importante como elemento primario en el comportamiento y por lo tanto en los daños de la albañilería.

Tabla 6. Estados límites de la norma propuesta para el diseño sísmico de edificios

\begin{tabular}{|l|l|l|c|}
\hline \multicolumn{1}{|c|}{ Estado límite } & \multicolumn{1}{c|}{ Definición del estado límite } & \multicolumn{1}{c|}{$\begin{array}{c}\text { Daño asociados a muros de } \\
\text { albañilería reforzada }\end{array}$} & $\begin{array}{c}\text { Distorsión } \\
\text { [\%] }\end{array}$ \\
\hline $\begin{array}{l}\text { Servicio } \\
\text { (PL1) }\end{array}$ & $\begin{array}{l}\text { Tratar de dejar la respuesta lejos del } \\
\text { punto de fluencia y controlar las } \\
\text { deformaciones para evitar daños no } \\
\text { estructurales. }\end{array}$ & $\begin{array}{l}\text {-Despreciable. } \\
\text {-Aparecen primeras grietas } \\
\text { apreciables a simple vista; } \\
\text {-Grietas diagonales delgadas; de } \\
\text { fácil reparación }\end{array}$ & $0.04-0.18$ \\
\hline $\begin{array}{l}\text { Operacional } \\
\text { (PL2) }\end{array}$ & $\begin{array}{l}\text { Define una respuesta dentro del } \\
\text { intervalo lineal, o con pequeñas } \\
\text { incursiones no lineales. }\end{array}$ & $\begin{array}{l}\text {-Grietas menores }(<3 \mathrm{~mm}) ; \text { no hay } \\
\text { deformaciones fuera del plano. } \\
\text {-Lesiones apreciables y algunos } \\
\text { deslizamientos en las junturas; } \\
\text { reparables }\end{array}$ & 0.62 \\
\hline $\begin{array}{l}\text { Daño controlado } \\
\text { (PL3) }\end{array}$ & $\begin{array}{l}\text { Es un estado difícil de evaluar } \\
\text { actualmente, pero se espera que los } \\
\text { daños sean bastante considerables, con } \\
\text { grietas visibles y algunos daños difícil } \\
\text { de reparar. }\end{array}$ & $\begin{array}{l}\text {-Grietas extensas (<6mm) } \\
\text { distribuidas a través del muro; } \\
\text { aplastamiento aislado. } \\
\text {-Lesiones considerables y } \\
\text { deslizamientos, roturas locales; } \\
\text { reparables con cierta dificultad. }\end{array}$ & 1.00 \\
\hline $\begin{array}{l}\text { Último } \\
\text { (PL4) }\end{array}$ & $\begin{array}{l}\text { En este estado se asegura que no se } \\
\text { producirá el colapso para el sismo } \\
\text { considerado, por lo que no interesa el } \\
\text { nivel de daño ni la magnitud de las } \\
\text { deformaciones. }\end{array}$ & $\begin{array}{l}\text {-Aplastamiento; grietas extensas; } \\
\text { daño alrededor de aberturas y } \\
\text { esquinas; caída de algunas } \\
\text { unidades. } \\
\text {-Pérdida notable de integridad; } \\
\text { reparación imposible }\end{array}$ & $<2.50$ \\
\hline
\end{tabular}

Tabla 7. Equivalencia entre estados límites

\begin{tabular}{|c|c|}
\hline Propuesta NCh433 & En este Trabajo \\
\hline PL1 & ELS/ ELO \\
\hline PL2 & ELDC \\
\hline PL3 & ELR \\
\hline PL4 & ELU \\
\hline
\end{tabular}

En cambio, si se considera el valor de la distorsión permisible ( $0.25 \%)$ recomendado por el anteproyecto de la norma mexicana de construcciones de mampostería confinada (NTCM2001, 2001) para revisar los desplazamientos laterales, éste valor representaría un estado de daño controlado (ELDC) para el 50\% de los muros de la muestra analizada en este trabajo. Del mismo modo, un valor de la distorsión permisible de entrepiso igual a un $0.4 \%$ para garantizar la seguridad contra el colapso de un edificio cuyo sistema estructural está formado por muros de albañilería confinada de piezas macizas o piezas huecas reforzada horizontalmente, propuesto en el anteproyecto de la norma mexicana de diseño por sismo (NTCS-2001, 2001), es razonable si se considera que sólo un $20 \%$ de los muros de la muestra alcanzarían un estado límite último (ELU), caracterizado por una reducción del $20 \%$ de su resistencia pero sin pérdida de su estabilidad antes cargas de tipo gravitacional al no haber daño severo de los elementos de confinamiento, situación que sólo se produce cuando las grietas del paño de albañilería penetran en los pilares. 
Los valores de la tabla 3 se obtuvieron para una muestra de muros de albañilería confinada construidos con unidades tradicionales (ladrillos cerámicos y bloques de hormigón) pero pueden aplicarse a muros construidos con otro tipo de unidades. Esta posibilidad queda confirmada si se consideran los resultados del estudio experimental realizado en muros de albañilería confinada construidos con unidades de materiales pumíticos (Urzúa et al.,2001). En la tabla 8 se comparan los valores promedios (fracción defectuosa de un 50\%) de las distorsiones angulares obtenidas de los ensayos realizados por Urzúa et al. (2001) con los propuestos en este trabajo para algunos de los estados límites.

Tabla 8. Comparación con los valores de las distorsiones angulares obtenidas de los ensayos realizados por Urzúa et al. (2001)

\begin{tabular}{|l|c|c|}
\hline \multicolumn{1}{|c|}{ ESTADOS LÍMITES } & Este trabajo & Urzúa et al. \\
\cline { 2 - 3 } & $\mathbf{5 0 \%}$ & Promedio \\
\hline Servicio (ELS) & 0.09 & 0.084 \\
\hline Operacional (ELO) & 0.13 & - \\
\hline Daño controlado (ELDC) & 0.26 & - \\
\hline Resistencia (ELR) & 0.40 & 0.41 \\
\hline Último (ELU) & 0.73 & 0.69 \\
\hline
\end{tabular}

\section{CONCLUSIONES}

A partir de un análisis de los resultados de estudios experimentales realizados en Chile, México y Venezuela, se ha podido establecer la capacidad de deformación de muros de albañilería confinada para distintos niveles de desempeño. Producto de este análisis, las conclusiones que se pueden destacar son las siguientes:

- Las distribuciones estadísticas que resultan para la distorsión angular asociada con diferentes estados límites o niveles de desempeño, permiten establecer las capacidades de deformación de la tabla 3 considerando tres valores distintos de la fracción defectuosa. Los valores de esta tabla son representativos de muros de albañilería confinada cuyo comportamiento está controlado por la falla de corte.

- Para los estados límites de servicio y operacional, la capacidad de deformación aumenta con la presencia de carga vertical. Cuando las cargas verticales actuantes producen tensiones normales mayores que $0.4 \mathrm{MPa}$, este aumento puede ser del orden de un $50 \%$ o más.

- La esbeltez es un factor importante en la capacidad de deformación de un muro de albañilería confinada, siendo su efecto más notorio en los estados límites asociado con los niveles de daños mayores. En particular, para el estado límite de resistencia la capacidad de deformación aumenta en la medida que aumenta la esbeltez del muro (Castilla, 1998). En general, es necesario reunir más resultados experimentales para cuantificar en forma confiable los efectos 
de la esbeltez del muro y de la presencia de armaduras de refuerzos horizontales en el paño de albañilería en la capacidad de deformación.

- En cuanto al efecto de la armadura horizontal de refuerzo, los resultados de los ensayos realizados en México permiten destacar que su efecto es claro para los estados límites de daño controlado, de resistencia y último, produciéndose un aumento de los niveles de deformación asociados con estos tres estados cuando la cuantía de refuerzo horizontal es mayor que un $0.05 \%$.

- Los valores propuestos en la tabla 3 son conservadores al considerar en conjunto los datos de los tres países de donde se obtuvo la información. Para realizar un análisis estadístico de las capacidades de deformación que represente las condiciones locales de cada país, es necesario enriquecer las bases de datos locales con más resultados de ensayos de muros de albañilería confinada.

- Los valores de las distorsiones de entrepiso asociados con los estados límites considerados en la propuesta de norma chilena de diseño sísmico son muy altos para ser utilizados en sistemas estructurados con muros de albañilería confinada.

- Los datos reunidos corresponden a muros de albañilería confinada construidos bajo condiciones de buen control de ejecución, propias de un estudio experimental. Las reducciones que puedan producirse en las capacidades de deformación por una falta de inspección no han sido cuantificadas.

\section{REFERENCIAS}

Alcocer, S, J Zepeda y M Ojeda (1997), "Estudio de factibilidad técnica del uso de tabique Vintex y Multex para viviendas económicas", Sistema Nacional de Protección Civil, Centro Nacional de Prevención de Desastres (CENAPRED), México D.F., México.

Aguilar, G (1997), "Efecto del refuerzo horizontal en el comportamiento de muros de mampostería confinada ante cargas laterales", Tesis de Licenciatura, Facultad de Ingeniería, Universidad Nacional Autónoma de México, México D.F., México.

Bonelli, P (1999), "Formato propuesta para la norma NCh433 y comentarios", Borrador de Trabajo.

Carrillo, V y A Molina (1997), "Evaluación del comportamiento de muros confinados de bloques macizos de arcilla con diferentes relaciones de aspecto ante cargas laterales alternantes", Trabajo para optar al Título de Ingeniero Civil, Universidad Central de Venezuela, Caracas, Venezuela.

Castilla, C (1998), "Evaluación de la respuesta de muros confinados de bloques de concreto contra ciclos severos de carga lateral", Tesis Doctoral, Universidad Central de Venezuela, Caracas, Venezuela.

Decanini, L, C Gavar, E D'Amore y P Bonelli (1993), “Control de daños en elementos no estructurales: experiencias y sugerencias", Sextas Jornadas Chilenas de Sismología e Ingeniería Antisísmica, Vol. II, Santiago, Chile, agosto. 
Díaz, R y R Vásquez (1993), “Comportamiento de muros de mampostería confinada reforzados horizontalmente", Tesis de Licenciatura, Facultad de Ingeniería, Universidad Nacional Autónoma de México, México D.F., México.

Diez, J (1987), "Estudio experimental de muros de albañilería sometidos a carga lateral alternada", Memoria para optar al título de Ingeniero Civil, Facultad de Ciencias Físicas y Matemáticas, Universidad de Chile, Santiago, Chile.

Gallegos, H (1989), Albañilería Estructural, Pontificia Universidad Católica del Perú, Fondo Editorial, Lima, Perú.

Herrera, E (1992), "Efecto de la carga vertical en el comportamiento de muros de albañilería reforzada sometidos a carga lateral alternada", Memoria para optar al título de Ingeniero Civil, Facultad de Ciencias Físicas y Matemáticas, Universidad de Chile, Santiago, Chile.

INN (1997), Norma Chilena NCh2123Of.97 - Albañilería confinada-Requisitos de diseño y cálculo, Instituto Nacional de Normalización, Santiago, Chile.

Moya, A (2002), "Estudio de los daños del terremoto de Chillán 1939”, Memoria para optar al título de Ingeniero Civil, Facultad de Ciencias Físicas y Matemáticas, Universidad de Chile, Santiago, Chile.

Muñoz, W (1992), "Estudio experimental del comportamiento de muros de albañilería de bloques de hormigón sometidos a carga lateral alternada", Memoria para optar al título de Ingeniero Civil, Facultad de Ciencias Físicas y Matemáticas, Universidad de Chile, Santiago, Chile.

NTCM-2001 (2001), Normas Técnicas Complementarias para Diseño y Construcción de Estructuras de Mampostería, Anteproyecto del Comité de Normas, mayo.

NTCS-2001 (2001), Normas Técnicas Complementarias para Diseño por Sismo, Anteproyecto del Comité de Normas, mayo.

Paulay, T y M J N Priestley (1991), Seismic design of reinforced concrete and masonry buildings, John Wiley \& Sons, Nueva York, Estados Unidos.

Sánchez, T (1998), "Comportamiento de estructuras de mampostería confinada sujetas a cargas laterales", Tesis de Maestría, División de Estudios de Posgrado de la Facultad de Ingeniería, Universidad Nacional Autónoma de México, México D.F., México.

Schmidt, A (2002), "Capacidad de deformación de muros de albañilería confinada. Antecedentes experimentales", Memoria para optar al título de Ingeniero Civil, Facultad de Ciencias Físicas y Matemáticas, Universidad de Chile, Santiago, Chile.

Urzúa, D A, R Padilla y R Loza (2001), "Influencia de la carga vertical en la resistencia sísmica de muros de albañilería confinada elaborados con materiales pumíticos típicos de Guadalajara" Memorias Técnicas, XIII Congreso Nacional de Ingeniería Sísmica, Guadalajara, Jalisco, México, octubre. 